\title{
Safe Disposal of Slaughter House Waste
}

\author{
Malav OP*, Birla R, Virk KS, Sandhu HS, Mehta N, Kumar P and Wagh RV \\ Department of Livestock products Technology, India
}

*Corresponding author: Om Prakash Malav, Department of Livestock products Technology, College of Veterinary Science, , Ludhiana, Punjab, India

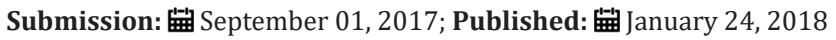

\begin{abstract}
Slaughter house waste or byproducts are to be disposed of or utilized appropriately so as to prevent contamination of the environment and the formation of valuable products which may increase the income of the abattoir and livestock owners. Both Solid and liquid wastes generated from the slaughter houses and animal processing plants having high nutritive value, therefore the Biochemical oxygen demand (BOD) is very high and these have high polluting potential when directly let into to the main water courses and environment. There are different methods for the treatment and processing of these wastes which reduces their polluting potential and these may be converted into useful products. The slaughter house byproducts consisting of heads, feet, offal, bone, hair, blood and feathers may be processed in rendering plants producing meat meal, bone meal, carcass meal, tallow and degreased bone. Efficient utilization of animal by-products has direct impact on the economy and environmental pollution of the country. Non-utilization or under-utilization of by-products not only lead to loss of potential revenues but also lead to the added and increasing cost of disposal of these products along with major aesthetic and serious health problems.
\end{abstract}

\section{Introduction}

Slaughter house wastes are any discarded materials generated during production and processing of meat from live animals, it may be solid, liquid, and semi-solid material. These wastes are to be disposed of appropriately so as to prevent contamination of the environment and the serious consequences such as spread of diseases, foul smells and unaesthetic view. A major part of the waste generated from the slaughter houses constitutes the Byproducts which are handled by the animal by-products industry to produce many valuable products such as leather, casings, catgut, bone meal, meat meal, gelatin, Neat's foot oil, ornamental items etc.

\section{Classification of Slaughter House Waste}

On the basis of usage as food, the byproducts can be categorized into edible and non-edible, however, some of the byproducts have medicinal values and classed under pharmaceutical category. Edible by products generally include all those organs which can be consumed viz. liver, lungs, heart, brain, intestine etc. They are also known as variety meats or 'fancy meat'. All those by products that cannot be consumed directly as food are non-edible byproducts e.g. hides, horns, ears, hooves, nails, bristles etc. All the condemned parts of animal carcass also fall in this category. This demarcation of edible and non-edible byproducts is not universally acceptable as it is largely dependent on customs, traditions, purchasing power, food choices etc. of consumers. In one region, consumption of a particular by product may be a taboo and in other region it may be a delicacy. The yield of edible by-products from animals varies tremendously depending on species, sex, live weight, fatness and methods of collection. In general, the total by-products range from
$10 \%$ to $30 \%$ of the live weight for beef, pork and lamb and from $5 \%$ to $6 \%$ of the live weight of chickens [1]. The yield of edible byproducts including blood and organs in cattle averages $12 \%$, in sheep $14 \%$, and, if pork rinds are also included, $14 \%$ for hogs. The consumption of offal is also influenced by disease outbreaks and government policies e.g. brain and spinal cord were out of the list for consumption since the outbreak of BSE (Mad cow disease). On the basis of live weight of animal the by-products account for almost $60 \%$ and out of this $40 \%$ are edible and $20 \%$ are inedible [2].

The byproducts can also be divided into primary and secondary by products. Primary by products also known as principal byproducts are harvested directly from the animals whereas the secondary byproducts are derived from principal byproducts e.g. Bones are primary by products whereas gelatin is secondary by product. The secondary byproducts have a huge market and yield large revenues. Another basis of classification of animal byproducts is their ultimate use. It includes agricultural byproducts (meat meal, fertilizers etc.), industrial by products (gelatin, glue etc.) and pharmaceutical byproducts such as hormones, bio-chemicals etc. [3].

On the basis of consistent of waste generated from the abattoir, it may be classified as solid and liquid waste. Solid waste includes edible and non-edible offal, hide and skin, dung, GIT contents, left over feed, hairs, bristles etc, whereas the liquid waste include effluent generated from slaughter houses which is the waste water originated from the different slaughtering and dressing operations. 


\section{Treatment of Solid Wastes from Slaughter Houses}

\section{Methods of handling viscera, paunch and intestines}

Viscera can be recovered as edible products (e.g. heart, liver, lung). They can also be separated for inedible rendering or processing. The paunch contents, 'paunch manure' (partially digested feed), is estimated to range from 27 to $40 \mathrm{~kg} / \mathrm{animal}$. The paunch can be handled in four ways: Total dumping- all of the paunch contents are flushed away into the sewer, wet dumpingthe paunch contents are washed out and the wet slurry is screened on the presence of gross solids, which are subsequently removed, dry dumping. The paunch contents are dumped for subsequent rendering or for disposal as solid waste without needless water flushing, whole paunch handling- the entire paunch may be removed, intact, for rendering or for disposal as solid waste. Intestines may be rendered directly, or hashed and washed prior to rendering.

\section{Bio gas production}

Due to the abundant biomass wastes generated by slaughterhouse, these biomass resources potential for biogas production by the process of biomethenation, this process use slaughterhouse waste for production of biogas. The success of the process, especially the effective removal BOD has led biogas plant to be acceptable for slaughter house [4]. Wastes consisting of rumen and paunch contents, dung, agriculture residue, fat and blood are processed in biomethenation plant. Power plants have been designed to produce biogas $(60 \%$ methane, $30 \%$ carbon dioxide and traces of hydrogen, carbon monoxide etc.) by digestion of animal waste [5]. The biogas can be used for boiler or power generation.

Large slaughter house are mostly located around cities and congested areas. They generate substantial quality of solid wastes, which have to be processed in environmentally acceptable manner. For the large slaughter houses, bio-methanation of waste is suggested. Bio-methanation requires less space, which is advantageous for the slaughter houses with land constraints. Bio gas produced from this operation may fulfill the energy requirements of slaughter unit and adjoining areas.

\section{Composting}

Composting involves the aerobic biological decomposition of organic materials to produce a stable humus-like product. The slaughter house waste can be used for compost making. The left over feed, dung from the lair age, ruminal and intestinal contents, blood, meat trimmings, floor sweepings, hair, feathers, hide trimmings can be stabilized by composting. It will produce very good quality bio-manure which may be utilized as fertilizers for the agriculture land and gardens.

\section{Rendering}

This is a useful method for the physical separation of fats from solids and water. All the animal matter such as inedible offal, tissues, meat trimmings, waste and condemned meat, bones etc. can be processed in a rendering system as the main constituents of animal matter are fat, water and solids. Rendering is affected by heating and rupturing connective tissue of individual fat and muscle cells so that raw fat and other material bound within is freed. In rendering, fat recovered is used for industrial purposes, such as soap and greases. Fat recovered from flesh of healthy parts can also be used for edible purposes. Solid portion, which is known as meat meal or bone meal, is utilized for the manufacture of stock feed and fertilizers. Rendering is carried out in dry rendering or wet rendering plants

\section{Effective utilization of slaughterhouse byproducts}

Efficient utilization of by-products has direct impact on the economy and environmental pollution of the country. Nonutilization or underutilization of by-products not only lead to loss of potential revenues but also lead to the added and increasing cost of disposal of these products. Non-utilization of animal by-products in a proper way may create major aesthetic and catastrophic health problems. Besides pollution and hazard aspects, in many cases meat, poultry and fish processing wastes have a potential for recycling raw materials or for conversion into useful products of higher value. Traditions, culture and religion are often important when a meat by-product is being utilized for food. By-products such as blood, liver, lung, kidney, brains, spleen and tripe have good nutritive value. Technologies have been developed for number of high value products like collagen sheets, tallow, keratin hydrolysates, ossein, pet foods, animal feeds etc. from different slaughter house byproducts. Other important product that can be prepared slaughter house byproducts include leather, carcass meal, rendered fat, sausage casings, blood meal, horn and hoof meal etc.

\section{Treatment of effluent - the liquid waste}

Effluent is the waste water generated from the meat processing plant. It has high polluting potential, which may causes degradation of the environment to a large extent. The wastewater produced contains a variety of organic and inorganic pollutants. For hygienic reasons, slaughter houses use large amount of water in processing operations (slaughtering, scalding and cleaning), which produces large amount of wastewater. The major environmental problem associated with this abattoir wastewater is the large amount of suspended solids and liquid waste as well as odour generation. Processes using ultra filtration-reverse osmosis, chemical precipitation-reverse osmosis and chemical precipitation-ultra filtration-reverse osmosis have been used in the treatment of meat industry wastewaters [6].

For the safe disposal of liquid and solid waste, the following action should be taken:

i. Separation of blood: The blood from slaughtered animals will coagulate into a solid mass, which may block up both open and closed drains. It is therefore recommended that the blood is collected and used for human consumption, stock feed production or fertilizers.

ii. Screening of solids: Solids (meat or skin trimmings, hair, 
pieces of bones, hooves, etc.) must be screened. This may be done by providing the drains with vertical sieves.

iii. Trapping of grease: Effluents from slaughterhouses always contain small amounts of fat (melted fat or small pieces of fatty tissues). Grease traps should be installed in the drains. The fat solidifies, rises to the surface and can be removed regularly.

iv. Biological treatment: Involves aerobic and anaerobic processes for the conversion of complex soluble and insoluble organic compounds into simple non-polluting compounds.

\section{Conclusion}

Proper treatment and efficient utilization of slaughter house waste and animal by-products respectively are the need of the hour since these have direct impact on the economy and environmental pollution of the world. Treatment of slaughter house wastes will lower down the chances of environmental pollution to many fold and efficient utilization of slaughter house byproducts will lead to the production many valuable products, useful for animal feed, industries, human feed, agriculture etc along with increasing the income of entrepreneurs and farmers.

\section{References}

1. Irshad A, Sureshkumar S, Shukoor AS, Sutha M (2015) Slaughter house by-product utilization for sustainable meat industry- a review. International Journal of Development Research 5(6): 4725-4734.

2. Chatli MK, Padda GS, Devatkal SK (2005) Augmentation of animal byproducts processing for the sustainability of meat industry. Indian Food Industry 24(5): 69-73.

3. Wani SA, Gazalli H, Sohi AH (2014) Animal Byproducts-Efficient utilization vis-à-vis benefits. In: Compendium of $6^{\text {th }}$ conference of Indian Meat Science Association and National Symposium on Sustainable meat production for nutritional security and consumer well-being: Challenges and strategies, 28-30 ${ }^{\text {th }}$ November, DUVASU, Mathura, India.

4. Hejnfelt A, Angelidaki I (2009) Anaerobic digestion of slaughterhouse by-products. Biomass Bioenergy 33(8): 1046-1054.

5. Sharma BD (2003) Modern abattoir practices and animal byproducts technology, Jaypee Brothers Medical Publishers (P) Ltd., New Delhi, India.

6. Bohdziewicz J, Sroka E (2005) Treatment of wastewater from the meat industry applying integrated membrane systems. Process Biochem 40:1339-1346. 